(C)2009 IEEE. Personal use of this material is permitted. However, permission to reprint/republish this material for advertising or promotional purposes or for creating new collective works for resale or redistribution to servers or lists, or to reuse any copyrighted component of this work in other works must be obtained from the IEEE. 


\title{
On the design of non-overshooting linear tracking controllers for right-invertible systems
}

\author{
Robert Schmid and Lorenzo Ntogramatzidis
}

\begin{abstract}
We consider the use of linear state feedback control to achieve a non-overshooting step response, for rightinvertible non-square multivariable systems. A method is given for designing a linear time-invariant state-feedback controller to asymptotically track a constant step reference with zero overshoot and arbitrarily small rise time, under some mild assumptions.
\end{abstract}

\section{INTRODUCTION}

The problem of ensuring that a linear time invariant (LTI) plant has a non-overshooting step response has been studied for the past few decades. The problem is of importance in several applications such as manufacturing processes, where overshoot can compromise tolerances and damage the product.

Some recent papers have considered the problem of designing a suitable closed-loop feedback controller to achieve a non-overshooting response. For continuous time singleinput single-output (SISO) systems, in [1] an eigenstructure assignment method is given to obtain a non-overshooting LTI state feedback controller for plants with one non-minimum phase zero. Stable non-minimum phase SISO systems are considered in [2], where the existence of an output feedback controller is proved to give a non-overshooting step response, provided the plant has no zeros on the imaginary axis. In [4] it is shown how to give two parameter feedback controller for an LTI plant that renders the step response non-overshooting. In [3] conditions are given for the existence of a controller to achieve a sign invariant impulse response, and hence also a non-overshooting step response. Corresponding conditions for discrete systems are given in [5]. A common feature of these recent papers [1]-[5] was that they considered only SISO systems, which were assumed to be initially at rest.

Recently [9] considered invertible stabilisable multipleinput multiple-output (MIMO) systems, and used linear statefeedback control to design a non-overshooting controller for a step reference. The design methods proposed there make use of the combined eigenvalue and eigenvector placement methods given in [8], and are applicable to both continuous time and discrete time systems. Moreover, the design method is applicable to both minimum phase and non-minimum phase systems. Conditions are given under which a linear state-feedback controller can be obtained to asymptotically track a step reference with guaranteed zero overshoot, from

R. Schmid is with the Department of Electrical and Electronic Engineering, The University of Melbourne, Parkville, Vic. 3010, Australia. E-mail: rschmideunimelb. edu. au

L. Ntogramatzidis is with the Department of Mathematics and Statistics, Curtin University of Technology, Perth, WA, Australia. E-mail: L.Ntogramatzidis@curtin.edu.au any initial condition. The controller can be readily chosen to achieve any desired convergence rate.

In this paper we continue the investigation of the tracking problem considered in [9], and consider non-invertible stabilisable MIMO systems, where the initial condition is not necessarily at zero. Non-invertible generically means the number of inputs and outputs of the plant are unequal. For right-invertible systems with fewer outputs than control inputs, we obtain a linear state-feedback controller that yields a non-overshooting step response, in all components of the output vector. The proposed control law achieves a non-overshooting response from all initial conditions, and the design scheme may be applied to minimum phase as well as non-minimum phase systems. Moreover, there is considerable freedom to choose the closed-loop poles. As such, the convergence rate can be chosen to be arbitrarily fast or slow, to satisfy any desired settling time or actuator constraints.

\section{Problem Formulation}

We consider the LTI system $\Sigma$ governed by

$$
\Sigma:\left\{\begin{array}{l}
\dot{x}(t)=A x(t)+B u(t), x(0)=x_{0}, \\
y(t)=C x(t)+D u(t),
\end{array}\right.
$$

where, for all $t \in \mathbb{T}, x(t) \in \mathbb{R}^{n}$ is the state, $u(t) \in \mathbb{R}^{m}$ is the control input, $y(t) \in \mathbb{R}^{p}$ is the output, and $A, B, C$ and $D$ are appropriate dimensional constant matrices. We assume $B$ and $\left[\begin{array}{ll}C & D\end{array}\right]$ are of full rank. As a standing assumption throughout the paper, we also assume that $\Sigma$ is stabilisable, i.e., it is either controllable, or else all uncontrollable modes lie in $\mathbb{C}^{-}$, the open left-half complex plane.

In this paper we are concerned with the problem of designing a state-feedback control law

$$
u(t)=F\left(x(t)-x_{\mathrm{ss}}\right)+u_{\mathrm{ss}},
$$

where $x_{\mathrm{ss}} \in \mathbb{R}^{n}$ and $u_{\mathrm{ss}} \in \mathbb{R}^{m}$ are solutions of

$$
\begin{aligned}
& 0=A x_{\mathrm{ss}}+B u_{\mathrm{ss}}, \\
& r=C x_{\mathrm{ss}}+D u_{\mathrm{ss}},
\end{aligned}
$$

such that the output $y(t)$, from a given initial state $x_{0}$, tracks a given step reference $r \in \mathbb{R}^{p}$ with zero steady-state tracking error and without overshoot. If suitable $F, x_{\mathrm{ss}}$ and $u_{\mathrm{ss}}$ exist for a given $r \in \mathbb{R}^{p}$ then we say that the step reference $r$ is trackable.

Let $P_{\Sigma}(\lambda):=\left[\begin{array}{cc}A-\lambda & I \\ C & D\end{array}\right]$ denote the system matrix pencil. We recall that system $\Sigma$ is right-invertible if and only if $\operatorname{rank} P_{\Sigma}(\lambda)=n+p$ for all but finitely many $\lambda \in \mathbb{C}$, 
and that $z_{0} \in \mathbb{C}$ is an invariant zero of $\Sigma$ if and only if rank $P_{\Sigma}\left(z_{0}\right)<$ normrank $P_{\Sigma}(\lambda)$. In the following lemma, the standard conditions are provided to ensure that asymptotic tracking can be achieved for all $r \in \mathbb{R}^{p}$ :

Lemma 2.1: Assume $\Sigma$ is right-invertible, stabilisable, and has no invariant zeros at the origin. Let $F$ be such that $A+B F$ is stable. Then every step reference $r \in \mathbb{R}^{p}$ is trackable with zero steady-state tracking error from any initial condition $x_{0} \in \mathbb{R}^{n}$ with the control law (2).

The tracking problem addressed in this paper is the one of choosing the gain matrix $F$ such that not only does the output $y(t)$ track the step reference $r$ with zero steady-state error, but reference tracking is achieved without overshoot in the output response. We formally define the non-overshooting property as follows:

Definition 2.1: (Non-overshooting step response)

Let $r \in \mathbb{R}^{p}$ be a trackable step reference. Then $\Sigma$ has a non-overshooting response for $r$ from the initial condition $x_{0} \in \mathbb{R}^{n}$ if the output $y(t)$ of (1) arising from $x_{0}$ yields a tracking error $\epsilon(t)=r-y(t)$ that satisfies

(i) $\epsilon(t)$ converges to zero as $t$ tends to infinity, and

(ii) $\epsilon(t)$ has no changes of sign in any component, i.e., for each $i \in\{1, \ldots, p\}, \operatorname{sgn}\left(\epsilon_{i}(t)\right)$ is constant for all $t \in \mathbb{T}$.

We say that $\Sigma$ has a globally non-overshooting response for $r$ if the output $y$ is non-overshooting for all initial conditions $x_{0}$.

The following lemma on eigenstructure assignment is essential to our design methods. It is easily derived from the classic eigenstructure assignment algorithm by B.C. Moore given in [8].

Lemma 2.2: [9] Let $\mathcal{L}=\left\{\lambda_{1}, \ldots, \lambda_{n}\right\} \in \mathbb{C}$ be a self conjugate set of $n$ distinct complex numbers. Let $\mathcal{S}=$ $\left\{s_{1}, \ldots, s_{n}\right\} \subset \mathbb{R}^{p}$ be a set of $n$ (not necessarily distinct) vectors in $\mathbb{R}^{p}$. Assume that, for each $i \in\{1, \ldots, n\}$, the matrix equation

$$
\left[\begin{array}{cc}
A-\lambda_{i} I & B \\
C & D
\end{array}\right]\left[\begin{array}{c}
v_{i} \\
w_{i}
\end{array}\right]=\left[\begin{array}{c}
0 \\
s_{i}
\end{array}\right]
$$

has solutions sets $\mathcal{V}=\left\{v_{1}, \ldots, v_{n}\right\} \subset \mathbb{C}^{n}$ and $\mathcal{W}=$ $\left\{w_{1}, \ldots, w_{n}\right\} \subset \mathbb{C}^{p}$, and that the set $\mathcal{V}$ is linearly independent. Then, a unique real feedback matrix $F$ exists such that, for all $i \in\{1, \ldots, n\}$,

$$
\begin{aligned}
& (A+B F) v_{i}=\lambda_{i} v_{i}, \\
& (C+D F) v_{i}=s_{i} .
\end{aligned}
$$

We note that Moore's algorithm can readily be executed with MATLAB $^{\circledR}$.

\section{DESIGN OF NON-OVERSHOOTING FEEDBACK CONTROLLERS WHEN $p<m$.}

In this section, we consider systems subject to the following assumption:

Assumption 3.1: System $\Sigma$ is such that $p<m$ and is right-invertible. Moreover, $\Sigma$ has no invariant zeros at the origin, and has at most $n-p$ distinct uncontrollable eigenvalues.
Since here it is assumed that the number of control inputs is greater than the number of controlled outputs, by Lemma 2.1 every $r \in \mathbb{R}^{p}$ is trackable. We now see how to use this additional control input to design a state feedback control law for the system to achieve a globally non-overshooting step response with any desired convergence rate (settling time). We begin by augmenting the system $\Sigma$ by adding one additional row vector $C_{p+1}$ and $D_{p+1}$ to matrices $C$ and $D$ as follows:

$$
\bar{C}=\left[\begin{array}{c}
C \\
C_{p+1}
\end{array}\right], \quad \bar{D}=\left[\begin{array}{c}
D \\
D_{p+1}
\end{array}\right] .
$$

This yields the augmented system

$$
\Sigma_{\text {aug }}:\left\{\begin{array}{l}
\dot{x}(t)=A x(t)+B u(t), \\
\bar{y}(t)=\bar{C} x(t)+\bar{D} u(t),
\end{array}\right.
$$

The row vectors $C_{p+1}$ and $D_{p+1}$ may be freely chosen provided that

- $\left[\begin{array}{ll}\bar{C} & \bar{D}\end{array}\right]$ has $p+1$ linearly independent rows;

- $\Sigma_{a u g}$ is right-invertible, and has no invariant zeros at the origin.

These properties may be ensured by choosing the additional row $\left[\begin{array}{ll}C_{p+1} & D_{p+1}\end{array}\right]$ to be linearly independent of all the rows of $\left[\begin{array}{ll}A & B \\ C & D\end{array}\right]$, which is possible because $p<m$. Then $\left[\begin{array}{cc}A & B \\ \bar{C} & \bar{D}\end{array}\right]$ has full row rank $n+p+1$, and thus $\Sigma_{a u g}$ has no invariant zeros at the origin. Since $\Sigma$ is right-invertible, this choice also guarantees that $\Sigma_{a u g}$ is right-invertible. The set of invariant zeros of $\Sigma_{a u g}$ includes the invariant zeros of $\Sigma$. Since $\Sigma$ is right-invertible, all its uncontrollable modes (which are stable) are also invariant zeros [9]. The same is true for $\Sigma_{a u g}$. However, since the set of uncontrollable modes of $\Sigma$ and $\Sigma_{a u g}$ are the same, and since both $\Sigma$ and $\Sigma_{a u g}$ are stabilisable, it follows that any additional invariant zeros introduced in $\Sigma_{a u g}$ via the introduction of the additional row $\left[\begin{array}{ll}C_{p+1} & D_{p+1}\end{array}\right]$ are not uncontrollable eigenvalues of the pair $(A, B)$. Furthermore, since $(A, B)$ has no more than $n-p$ uncontrollable eigenvalues, $\Sigma_{a u g}$ has no more than $n-p$ uncontrollable eigenvalues that are also invariant zeros.

We may now use $\Sigma_{\text {aug }}$ to design a control law for $\Sigma$ as follows. Let $\mathcal{L}=\left\{\lambda_{1}, \ldots, \lambda_{n}\right\} \subset \mathbb{C}$ denote the set of distinct stable closed-loop eigenvalues of $A+B F$ to be chosen. For $i \in\{1, \ldots, p\}$, these may be any distinct real stable modes; however these modes must not include any zeros of $\Sigma_{a u g}$. If $\Sigma$ is controllable, the remaining modes $\lambda_{p+1}, \ldots, \lambda_{n}$ may be freely chosen to be any set of distinct stable complex numbers; these modes are permitted to include zeros of $\Sigma_{\text {aug }}$, with the only requirement that $\mathcal{L}$ be self-conjugate. If $\Sigma$ has uncontrollable modes, the choice of $\mathcal{L}$ must be modified as follows: all uncontrollable modes of $\Sigma$ (which are asymptotically stable) must be included in $\mathcal{L}$. As $\Sigma_{\text {aug }}$ is right-invertible, these modes are also zeros of $\Sigma_{a u g}$, and hence these modes must be counted among the modes $\lambda_{p+1}, \ldots, \lambda_{n}$. Since the first $p$ modes of $\mathcal{L}$ must not include zeros of $\Sigma_{\text {aug }}, \mathcal{L}$ can have at most $n-p$ modes that are also zeros of $\Sigma_{\text {aug }}$. This is why Assumption 3.1 
requires $\Sigma$, and hence $\Sigma_{\text {aug }}$, to have at most $n-p$ distinct uncontrollable eigenvalues.

Let $\left\{\bar{e}_{1}, \ldots, \bar{e}_{p+1}\right\}$ be the canonical basis of $\mathbb{R}^{p+1}$, and $\mathcal{S}=\left\{s_{1}, \ldots, s_{n}\right\} \subset \mathbb{R}^{p+1}$ be such that

$$
s_{i}=\left\{\begin{array}{cl}
\bar{e}_{i} & \text { for } i \in\{1, \ldots, p\} \\
\bar{e}_{p+1} & \text { for } i \in\{p+1, \ldots, n\} \\
& \text { if } \lambda_{i} \text { is not a zero of } \Sigma_{a u g} \\
0 & \text { for } i \in\{p+1, \ldots, n\} \\
& \text { if } \lambda_{i} \text { is a zero of } \Sigma_{a u g}
\end{array}\right.
$$

Solving the matrix equation

$$
\left[\begin{array}{cc}
A-\lambda_{i} I & B \\
\bar{C} & \bar{D}
\end{array}\right]\left[\begin{array}{c}
v_{i} \\
w_{i}
\end{array}\right]=\left[\begin{array}{c}
0 \\
s_{i}
\end{array}\right]
$$

for the elements $s_{i}$ in $\mathcal{S}$ for all $i \in\{1, \ldots, n\}$, we obtain the sets of vectors $\mathcal{V}=\left\{v_{1}, \ldots, v_{n}\right\} \subset \mathbb{C}^{n}$ and $\mathcal{W}=$ $\left\{w_{1}, \ldots, w_{n}\right\} \subset \mathbb{C}^{p+1}$. Note that (11) is solvable for all $\lambda_{i}$ and all $s_{i}$ in view of the right-invertibility of $\Sigma_{a u g}$, which guarantees that when $\lambda_{i}$ is not an invariant zero of $\Sigma_{a u g}$, the Rosenbrock matrix $P_{\Sigma_{\text {aug }}}\left(\lambda_{i}\right)$ on the left-hand side of (11) is full row-rank. Provided the resulting $\mathcal{V}$ is linearly independent, by Lemma 2.2, the Moore algorithm can be applied to obtain a feedback gain matrix $F$ such that $A+B F$ has distinct eigenvalues and eigenvectors given by $\mathcal{L}$ and $\mathcal{V}$, respectively. Our next theorem shows that, for any $r \in \mathbb{R}^{p}$, this matrix $F$ can be used to obtain a state-feedback control law that yields a closed-loop system response converging to the step reference $r$ that is globally non-overshooting.

Theorem 3.1: Assume that $\Sigma$ satisfies Assumption 3.1. Let $r \in \mathbb{R}^{p}$, and let $F$ be obtained as above. Then, applying the state-feedback control law $u(t)$ given in (2) to $\Sigma$ yields a globally non-overshooting response for $r$.

Proof: Introduce the new state coordinates $\xi:=x-x_{\mathrm{ss}}$. Let $x_{0} \in \mathbb{R}^{n}$ be any initial condition. Applying the feedback law $u$ to $\Sigma$ we obtain the homogeneous system

$$
\Sigma_{h o m}:\left\{\begin{array}{l}
\dot{\xi}(t)=(A+B F) \xi(t), \\
y(t)=(C+D F) \xi(t)+r .
\end{array}\right.
$$

For any initial condition $\xi_{0} \in \mathbb{R}^{n}$, the tracking error $\epsilon(t)=$ $r-y(t)$ is given by $\epsilon(t)=-(C+D F) e^{(A+B F) t} \xi_{0}$. As the eigenvectors in $\mathcal{V}$ are linearly independent, the matrix $V:=\left[\begin{array}{llll}v_{1} & v_{2} & \ldots & v_{n}\end{array}\right]$ is invertible. Introduce $\alpha:=$ $\left[\begin{array}{llll}\alpha_{1} & \alpha_{2} & \ldots & \alpha_{n}\end{array}\right]^{\top}=V^{-1} \xi_{0}$. By (7) of Lemma 2.2 the matrix $F$ defined above is such that $(\bar{C}+\bar{D} F) v_{i}=s_{i}$, where $s_{i}$ is given in (10). Hence

$$
(C+D F) v_{i}=\left\{\begin{array}{cl}
e_{i} & \text { for } i \in\{1, \ldots, p\}, \\
0 & \text { for } i \in\{p+1, \ldots, n\},
\end{array}\right.
$$

where $e_{i}$ and 0 are vectors in $\mathbb{R}^{p}$, and so the tracking error is given by

$$
\begin{aligned}
\epsilon(t) & =-\sum_{i=1}^{n}(C+D F) v_{i} \alpha_{i} e^{\lambda_{i} t} \\
& =-\sum_{i=1}^{p} e_{i} \alpha_{i} e^{\lambda_{p} t} \\
& =-\left[\alpha_{1} e^{\lambda_{1} t} \ldots \alpha_{p} e^{\lambda_{p} t}\right]^{\top}
\end{aligned}
$$

As the eigenvalues all lie in $\mathbb{C}^{-}$, the system $\Sigma_{h o m}$ is asymptotically stable, and $\epsilon(t)$ converges to zero as $t$ tends to infinity. As each of the $p$ components of $\epsilon(t)$ contains exactly one mode, $\epsilon(t)$ does not change sign in any component, and $y(t)$ converges to $r$ without overshoot. As $x_{0}$ is arbitrary, $\Sigma$ has a globally non-overshooting response for $r$.

Remark 3.1: For any given choice of $\mathcal{L}$ and $\mathcal{S}$, it is not assured that the vectors in $\mathcal{V}$ obtained from solving (11) are linearly independent. To solve this problem we may use any of the $p$ ! possible ways to re-order the first $p$ canonical basis vectors in $\mathcal{S}$ and again solve (11) to obtain a new $\mathcal{V}$. If this failed to obtain a linearly independent $\mathcal{V}$, we may instead alter one of the eigenvalues in $\mathcal{L}$ and again solve (11) until a linearly independent set $\mathcal{V}$ is obtained. Note also that only modes $\lambda_{i}$ for $i \in\{1, \ldots, p\}$ are visible at the output. Hence, these may be chosen to obtain any desired rate of convergence of the output trajectory. Moreover, the convergence rate in the $i$-th output component is determined by $\lambda_{i}$. As the remaining $n-p$ modes do not affect the output, these may be chosen with a view to minimising control effort. If $\Sigma$ has some stable open-loop eigenvalues, we may include these among the non-visible closed-loop poles in $\mathcal{L}$, as control effort is minimised by reducing the shifting in the open-loop poles. Note also that $F$ is independent of both $r$ and $x_{0}$. Hence the control law is globally non-overshooting for all $r \in \mathbb{R}^{p}$. The values of $r$ and $x_{0}$ enter the control law $u$ only through the values of $x_{\mathrm{ss}}$ and $u_{\mathrm{ss}}$.

\section{EXAMPLES}

Example 4.1: Consider the MIMO system $\Sigma_{1}=$ $(A, B, C, D)$ with

$$
\begin{array}{cc}
A=\left[\begin{array}{cccc}
0 & 0 & 5 & 0 \\
0 & -1 & 0 & 0 \\
-1 & 0 & -6 & 3 \\
-6 & 0 & 3 & -3
\end{array}\right] \quad B=\left[\begin{array}{ccc}
-3 & 0 & 0 \\
0 & 0 & 0 \\
0 & 0 & -3 \\
-4 & 10 & 0
\end{array}\right] \\
C=\left[\begin{array}{cccc}
0 & 0 & 0 & 0 \\
-2 & -7 & 0 & 3
\end{array}\right] \quad D=\left[\begin{array}{ccc}
0 & 7 & -7 \\
0 & 0 & -2
\end{array}\right] .
\end{array}
$$

Hence, $\Sigma_{1}$ is a non-square right-invertible system with $n=4, p=2$ and $m=3$. The system has open-loop poles at $-0.116 \pm 3.46 i,-8.77$ and -1 . The stable pole at -1 is uncontrollable. Also, the system is of non-minimum phase with invariant zeros at $12.3,-6.3$, and -1 . Thus $\Sigma_{1}$ satisfies Assumption 3.1. Let assume the desired step reference is $r=\left[\begin{array}{ll}3-3 & -3\end{array}\right]^{\top}$. Following the design procedure outlined above, we first augment $C$ and $D$ as follows:

$$
\bar{C}=\left[\begin{array}{cccc}
0 & 0 & 0 & 0 \\
-2 & -7 & 0 & 3 \\
1 & 0 & 0 & 0
\end{array}\right], \quad \bar{D}=\left[\begin{array}{ccc}
0 & 7 & -7 \\
0 & 0 & -2 \\
0 & 0 & 0
\end{array}\right]
$$

such that the augmented system $(A, B, \bar{C}, \bar{D})$ remains rightinvertible. We next choose closed-loop poles $\left\{\lambda_{1}, \ldots, \lambda_{4}\right\}$ as follows: Since $\lambda_{1}$ and $\lambda_{2}$ are visible at the output, we may choose these for rapid convergence; they may be any real stable modes that are distinct from the zeros of $\Sigma_{1}$. Modes 
$\lambda_{3}$ and $\lambda_{4}$ are invisible at the output, so we may choose one of the stable open-loop poles, to minimise control effort. Lastly, we are forced to choose the uncontrollable mode at -1 , which is also a zero of the augmented system. A suitable selection is $\mathcal{L}=\{-4,-3,-8.77,-1\}$. Solving (11) for the vectors $\mathcal{S}=\left\{\left[\begin{array}{lll}1 & 0 & 0\end{array}\right]^{\top},\left[\begin{array}{lll}0 & 1 & 0\end{array}\right]^{\top},\left[\begin{array}{lll}0 & 0 & 1\end{array}\right]^{\top},\left[\begin{array}{lll}0 & 0 & 0\end{array}\right]^{\top}\right\}$, we obtain the linearly independent closed-loop eigenvectors $\mathcal{V}=\left\{v_{1}, \ldots, v_{4}\right\}$ given by

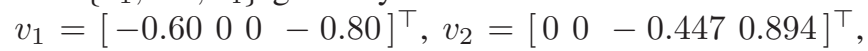
$v_{3}=\left[\begin{array}{llll}0 & 0 & 0.729 & 0.685\end{array}\right]^{\top}, v_{4}=\left[\begin{array}{llll}0 & 0.294 & 0.350 & 0.890\end{array}\right]^{\top}$. Applying Lemma 2.2 to $\mathcal{L}$ and $\mathcal{V}$ yields the gain matrix

$$
F=\left[\begin{array}{cccc}
2.9230 & 0 & 1.6667 & 0 \\
1.043 & 0.779 & 0.303 & -0.032 \\
-0.485 & -1.408 & -0.773 & 1.113
\end{array}\right]
$$

Applying the control law (2) with this $F$ to $\Sigma_{1}$ yields outputs that satisfy

$(C+D F) v_{1}=\left[\begin{array}{ll}1 & 0\end{array}\right]^{\top},(C+D F) v_{2}=\left[\begin{array}{ll}0 & 1\end{array}\right]^{\top}$,

$(C+D F) v_{3}=\left[\begin{array}{ll}0 & 0\end{array}\right]^{\top},(C+D F) v_{4}=\left[\begin{array}{ll}0 & 0\end{array}\right]^{\top}$.

Thus, both output components are driven only by a single exponential, and hence cannot overshoot. If we wish to increase the convergence speed, we may instead choose the first two eigenvalues to have larger modulus of the real part, e.g., $\mathcal{L}=\{-6,-5,-8.77,-1\}$. The system responses for these two sets of closed-loop eigenvalues are shown in Figures 1 and 2, for several different values of the initial condition $x_{0}$. In all cases, both components of the output trajectory converge to their target value without overshoot.

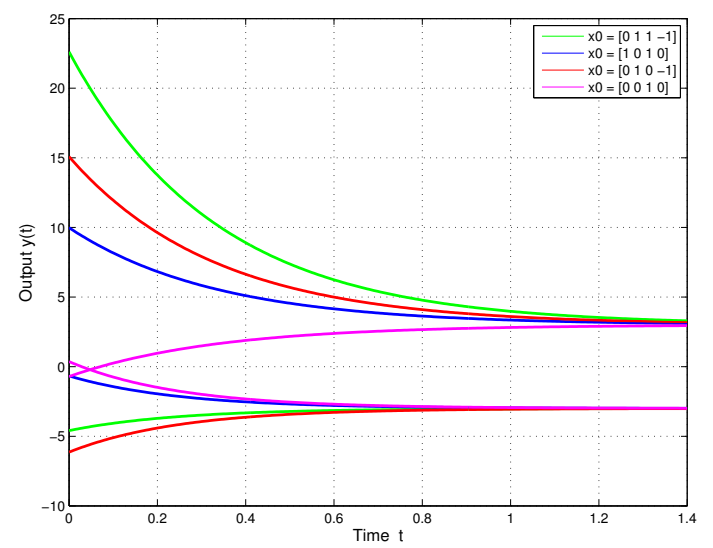

Fig. 1. MIMO system $\Sigma_{1}$ step response using Moore algorithm and closed loop poles $\mathcal{L}=\{-4,-3,-8.77,-1\}$.

We compared our control scheme against the transient performance achievable from the MIMO eigenvalue assignment scheme offered in [6], and which may be readily implemented with the MATLAB ${ }^{\circledR}$ command place. The system response for $\Sigma_{1}$ with closed loop poles $\mathcal{L}=$ $\{-6,-5,-8.77,-1\}$ is shown in Figure 3. We note that some components overshoot, and the convergence is much slower as the slow uncontrollable mode at $\lambda_{4}=-1$ remains visible at the output.

To compare the amplitude of the control effort employed in our approach against that of [6], we considered the control

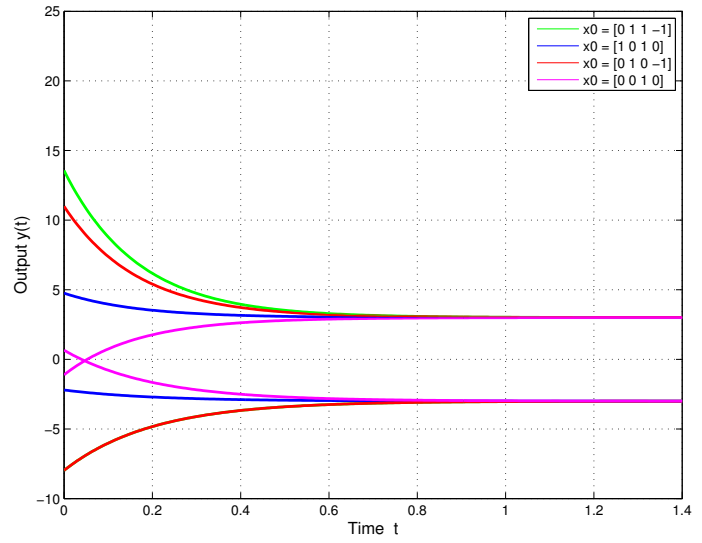

Fig. 2. $\Sigma_{1}$ step response using Moore algorithm and closed loop poles $\mathcal{L}=\{-6,-5,-8.77,-1\}$.

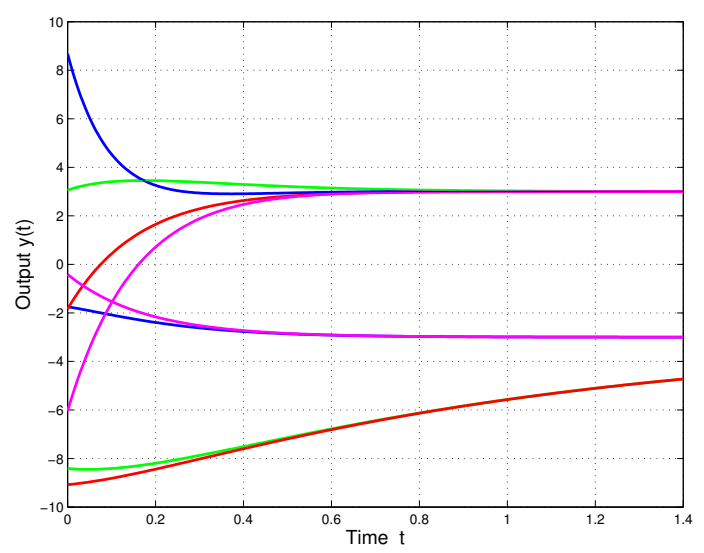

Fig. 3. $\Sigma_{1}$ step response using place algorithm and closed loop poles $\mathcal{L}=\{-6,-5,-8.77,-1\}$.

index

$$
\alpha(t)=\frac{\left|u_{N}(t)\right|}{\left|u_{P}(t)\right|}
$$

where $u_{N}$ is given in (2), and $u_{P}=F_{P}\left(x(t)-x_{\mathrm{ss}}\right)+u_{\mathrm{ss}}$ where $F_{P}$ is the feedback gain matrix obtained from the place command. Figure 4 shows the value of this control strength index; as the index is always less than unity, our control law uses less control effort than the one given in [6], for this simulation.

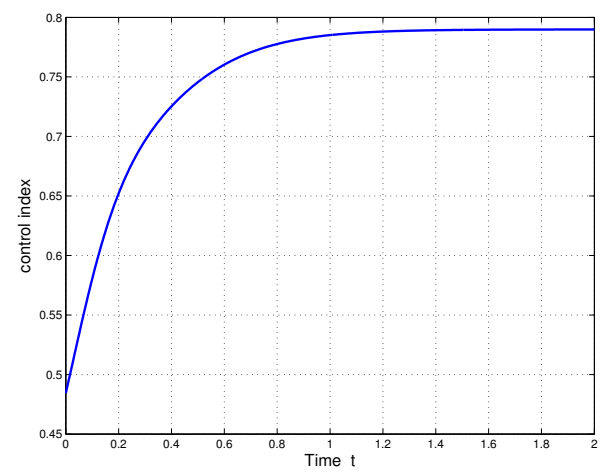

Fig. 4. Control strength index for MIMO system $\Sigma_{1}$. 
Example 4.2: The system $\Sigma_{2}=(A, B, C, D)$ with

$$
\begin{gathered}
A=\left[\begin{array}{ccc}
-3 & -1.5 & -0.5 \\
2 & 0 & 0 \\
0 & 1 & 0
\end{array}\right] \quad B=\left[\begin{array}{l}
2 \\
0 \\
0
\end{array}\right] \\
C=\left[\begin{array}{lll}
0.5 & 0.75 & 0.5
\end{array}\right] \quad D=[0]
\end{gathered}
$$

is a minimal state space realization of the transfer function

$$
P(s)=\frac{(s-1)(s-2)}{(s+1)^{3}}
$$

that was considered in Example 1 of [10]. $\Sigma_{2}$ is a strictly proper SISO non-minimum phase system with invariant zeros at $s=1$, and $s=2$. The transient response of $\Sigma_{2}$ is of particular interest because [7] showed that the step response of a strictly proper transfer function with one real positive zero must exhibit undershoot, and recently [10] showed that the step response of a strictly proper transfer function with two real positive zeroes must exhibit both undershoot and overshoot, if the settling time is sufficiently small.

As our results are for non-square systems, we consider the transient performance that may be achieved if it is possible to apply an additional control input for the plant. Thus we consider the enhanced system $\Sigma_{2 e}=\left(A, B_{e}, C, D_{e}\right)$ with

$$
B_{e}=\left[\begin{array}{ll}
2 & 0 \\
0 & 0 \\
0 & 1
\end{array}\right], \quad D_{e}=\left[\begin{array}{ll}
0 & 0
\end{array}\right]
$$

Then $\Sigma_{2 e}$ is a nonminimum phase right-invertible system with $n=3, m=2, p=1$ and an invariant zero at $s=3$. Using the above controller design, we are able to design feedback gain matrices to achieve a step response with any desired convergence rate. The step response of $\Sigma_{2 e}$ from zero initial conditions is shown for the three convergence rates $\lambda_{1}=-5, \lambda_{1}=-10$, and $\lambda_{1}=-50$ in Figure 5. In all cases, the output trajectory converges to the target value $r=1$ without undershoot or overshoot.

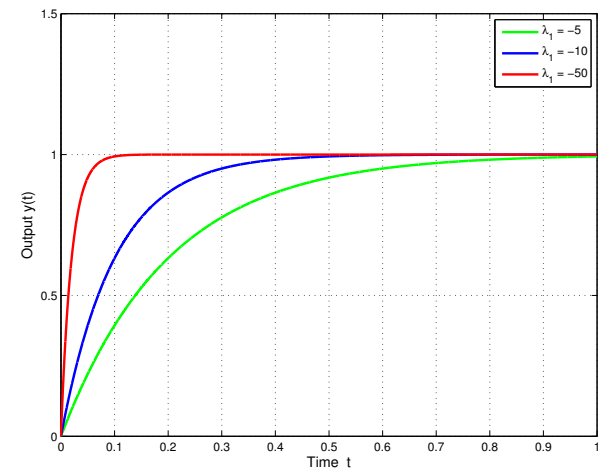

Fig. 5. MIMO system $\Sigma_{2 e}$ step response.

\section{CONCLUSION}

We have introduced new linear state feedback controller design methods to obtain a non-overshooting step response, for non-invertible MIMO systems. The methods complement those given in our earlier paper [9] for invertible MIMO systems.

\section{REFERENCES}

[1] M. Bement and S. Jayasuriya, "Use of state feedback to achieve a nonovershooting step response for a class of nonminimum phase systems", Journal of Dynamical Systems, Measurement and Control, vol. 126, pp. 657-660, 2004.

[2] M. Bement and S. Jayasuriya, "Construction of a set of nonovershooting Tracking controllers", Journal of Dynamical Systems, Measurement and Control, vol. 126, pp. 558-567, 2004.

[3] S. Darbha, "On the synthesis of controllers for continuous time LTI systems that achieve a non-negative impulse response", Automatica, vol. 39, pp. 159-165, 2003.

[4] S. Darbha and S.P. Bhattacharyya, "On the synthesis of controllers for a nonovershooting step response", IEEE Transactions on Automatic Control, vol. 48, pp. 797-799, 2003.

[5] S. Darbha and S.P. Bhattacharyya, "Controller synthesis for sign invariant impulse response", IEEE Transactions on Automatic Control, vol. 47, pp. 1346-1351, 2002.

[6] J. Kautsky, J. and N.K. Nichols, "Robust Pole Assignment in Linear State Feedback," International Journal of Control, vol. 41, pp. 11291155, 1985.

[7] R.H. Middleton, "Trade-offs in linear control system design", Automatica, 27 (1991), pp. 281-292.

[8] B.C. Moore, "On the Flexibility Offered by State Feedback in Multivariable systems Beyond Closed Loop Eigenvalue Assignment", IEEE Transactions on Automatic Control, pp. 689-692, 1976.

[9] R. Schmid and L. Ntogramatzidis, "Nonovershooting linear multivariable state feedback tracking controllers", Proceedings 17th IFAC World Congress, Seoul, 2008.

[10] J. Stewart and D.E. Davison, "On Overshoot and Nonminimum phase Zeros", IEEE Transactions on Automatic Control, 51 (2006), pp. $1378-1382$. 